\title{
Recombinant Interleukin-8
}

National Cancer Institute

\section{Source}

National Cancer Institute. Recombinant Interleukin-8. NCI Thesaurus. Code C1289.

A member of the CXC chemokine family. This chemokine is one of the major mediators of the inflammatory response. This chemokine is secreted by several cell types including monocytes, macrophages, T-lymphocytes, fibroblasts, endothelial cells, and keratinocytes. It functions as a chemoattractant, and is also a potent angiogenic factor. (from LocusLink) 